\title{
Applying research to practice: Generalist and specialist (visual ergonomics) consultancy
}

\author{
Jennifer Long ${ }^{\mathrm{a}, \mathrm{b}^{*}}$ and Airdrie Long ${ }^{\mathrm{c}}$ \\ ${ }^{\mathrm{a}}$ Jennifer Long Visual Ergonomics, PO Box 645 Katoomba NSW 2780, Australia \\ ${ }^{\mathrm{b}}$ School of Optometry and Vision Science, Rupert Myers Building North Wing, University of New South Wales \\ Sydney NSW 2052, Australia \\ ${ }^{\mathrm{C}}$ Considered Solutions, 5 The Crescent, Chatswood NSW 2067, Australia
}

\begin{abstract}
Ergonomics is a holistic discipline encompassing a wide range of special interest groups. The role of an ergonomics consultant is to provide integrated solutions to improve comfort, safety and productivity. In Australia, there are two types of consultants - generalists and specialists. Both have training in ergonomics but specialist knowledge may be the result of previous education or work experience. This paper presents three projects illustrating generalist and specialist (visual ergonomics) consultancy: development of a vision screening protocol, solving visual discomfort in an office environment and solving postural discomfort in heavy industry. These case studies demonstrate how multiple ergonomics consultants may work together to solve ergonomics problems. It also describes some of the challenges for consultants, for those engaging their services and for the ergonomics profession, e.g. recognizing the boundaries of expertise, sharing information with business competitors, the costs-benefits of engaging multiple consultants and the risk of fragmentation of ergonomics knowledge and solutions. Since ergonomics problems are often multifaceted, ergonomics consultants should have a solid grounding in all domains of ergonomics, even if they ultimately only practice in one specialty or domain. This will benefit the profession and ensure that ergonomics remains a holistic discipline.
\end{abstract}

Keywords: Professionalism, business, expertise, vision, problem-solving

\section{Introduction}

Ergonomics is a holistic discipline with three domains of interest: physical, cognitive and organizational ergonomics [6]. International Ergonomics Association (IEA) technical committees, or special interest groups cater for members with specific workplace or research interests and enable members to network and collaborate with like-minded people.

There are currently 26 technical committees within the IEA; these can be divided into several categories including industry-specific (e.g. computers, process control), population-specific (e.g. aging, children) and human-specific (e.g. auditory ergonomics, visual ergonomics). This means that there are potentially at least 26 different areas in which ergonomists can specialize.
Is it a contradiction to have specialties within a holistic discipline like ergonomics?

The development of specialties may simply reflect how a broadening knowledge base is managed by individuals within the profession. This concept was debated in medicine more than a century ago [11] and has been reported as a helpful strategy for practitioners trying to keep up to date with advancing knowledge [16]. Opponents to the formation of specialties may claim that there are simply a variety of applications within a field [15] although others argue that segments (and collegiality) within a group are important for the development of new knowledge and practice [10]. Nevertheless, there appears to be agreement that even if segments within a profession practice in different ways, individuals need to have a common training, or knowledge base, to understand and appreciate the various facets of their profession.

*Corresponding author. E-mail: jlong@visualergonomics.com.au, Telephone: +61 247823472 
In Australia, ergonomics consultants work within industry as company employees or are engaged as external consultants. One of the roles of an ergonomist is to identify possible causes of a problem, accept/reject these as plausible explanations and then deliver a solution to improve comfort, safety and productivity. This process is similar to that used by healthcare professionals [21] and other investigative practitioners [5] and is dependent on the ergonomist having a good working knowledge of physical, cognitive and organizational ergonomics, an up-to-date knowledge of advances in current research and an awareness of all the possible options to test. This holistic approach is defined as a core competency by the IEA [7].

Two types of ergonomics consultants exist in Australia: generalists and specialists. To achieve professional certification, both must have general training in ergonomics encompassing the three domains of interest in ergonomics [3] but specialist knowledge may be the result of previous education or workexperience in a particular industry. The majority of consultants work in micro-businesses (i.e. businesses employing less than 5 people [1]) but may work together in teams or be engaged on an individual basis, depending on the scope of a project.

The advantage of employing a generalist is that they should be able to apply research from many disciplines to solve any presenting ergonomics issue. However, their effectiveness may be limited by lack of in-depth knowledge on specific topics and this can have an impact on their ability to identify the source of a problem, offer the most appropriate solution or implement necessary modifications.

Specialists have evolved out of a need to solve complex issues which may be beyond the expertise of a generalist, in a similar way to the evolution of specialists within medicine [11]. Their strength lies in applying their particular expertise across a range of industries, tasks and/or populations. However, their effectiveness may be limited by exposure to only a small or specific aspect of a larger problem. This can make it difficult for them to offer a contextual solution which is appropriate and acceptable to the workplace.

\subsection{Aim}

The purpose of this paper is to present case examples of specialist and generalist ergonomics practice and then discuss some of the challenges for consul- tants, for those engaging consultancy services and for the ergonomics profession.

\subsection{Scope}

Visual ergonomics is used in this paper as the specialist case example. This was selected on the basis of the experience of both authors, one of whom works as a specialist (JL) and the other who works as a generalist (AL).

\subsection{Visual ergonomics}

Visual ergonomics is an example of humanspecific sub-category of ergonomics which draws on physical ergonomics (e.g. lighting, visual displays, workstation design, visual disabilities) and cognitive ergonomics (e.g. information design) and requires an underlying knowledge of the function of the visual system and of visual perception. Visual ergonomics has applications in many industries including transport, healthcare and manufacturing, for different population subgroups e.g. ageing workforce, children, people with visual disabilities and for visually demanding tasks e.g. visual displays, driving, assembly work.

\section{Methods}

Three case examples are presented in this paper:

- Development of a vision screening protocol in a manufacturing plant

- Solving visual discomfort in an office environment

- Solving postural discomfort in a heavy industry environment.

The case examples were selected on the basis that they illustrate:

- different facets of visual ergonomics

- issues facing specialist and generalist ergonomics consultants

- the role of specialist and generalist ergonomists in providing a holistic problem solving approach, even when the presenting problem appears to have a narrow focus.

They represent typical issues encountered by ergonomics consultants in Australia. For confidentiality reasons, the case examples have been de-identified. 


\section{Case examples}

\subsection{Development of a vision screening protocol for a manufacturing plant}

A vision screening program was requested by the management of a manufacturing plant to ensure that workers had adequate vision to perform their tasks. Vision screening protocols exist for some industries and tasks e.g. driving [8] but the unique nature of work in this factory meant that a vision screening protocol needed to be developed specifically for this workplace.

Although the company already employed a generalist ergonomist who had identified the need for the vision screening program, specialist knowledge was required to conduct a task analysis and identify vision specific tasks. Vision critical components were measured (e.g. sizes, working distances) and described (e.g. colour, layout) for later analysis. These components were then mapped to clinical tests and a pass/fail criteria developed based on visual reserves [17]. For example, if a worker needed to be able to read text $3 \mathrm{~mm}$ high, then for visual comfort they should be capable of reading text half this size, i.e. $1.5 \mathrm{~mm}$ high. This equates to reading text size $\mathrm{N} 8$ (printers' "points") on a near letter chart [9], so in this case, being able to read N8 would be set as the pass/fail criteria.

While it might have been possible for a subject matter expert (e.g. an optometrist or ophthalmologist without ergonomics training) to prescribe clinical tests suitable for a vision screening program based on measurements or details provided by the generalist ergonomist, the specialist ergonomist gained valuable information which assisted in the development and implementation of the program by adopting a consultative approach. For example:

- Understanding the intrinsic task difficulty was enhanced by the specialist ergonomist sitting with various employees during the course of their working day, observing the tasks, and attempting to perform the task herself. This knowledge also assisted the specialist select appropriate clinical tests for the vision screening program.

- Ensuring that the vision screening program was relevant to the workplace and integrating it with workplace policies and current legislation (e.g. Australian privacy legislation [4]) was achieved by consultation between the specialist, management and the generalist ergonomist.

\subsection{Solving visual discomfort in an office environment}

Within weeks of installing a new computer system (software and hardware), office workers began to report headaches and "eyestrain" which they attributed to the font size displayed on their computer monitors. A general ergonomics assessment had been conducted by occupational health and safety personnel within the company. One of their recommendations was for a "visual ergonomics assessment" to determine the validity of the office workers claims.

Analysis of the old and new technology revealed that text on the new display was $1 \mathrm{~mm}$ smaller than on the old display. Workers were observed to lean over their desk to view their work, consistent with predicted viewing distances for this size font [14]. Therefore it was conceivable that the increased visual demands of the small size font together with awkward postures were contributing to reports of headaches and eyestrain.

Interviews were conducted with some of the workers to better understand their difficulties with a view to devising an appropriate solution which would be acceptable to both the workers and management. It was then discovered that there were broader issues beyond the original scope of investigation. For example:

- Although workers could increase the font size with a zoom function on the screen page, this resulted in more mouse scrolling to view data.

- The increased mouse scrolling increased the time required to navigate screen pages. This was a source of frustration, particularly since workers operated to a tight time schedule.

- The increased mouse scrolling also posed a potential risk of musculoskeletal discomfort for workers.

- Lack of consultation with workers prior to implementation was a source of dissatisfaction. Many workers believed that consultation prior to implementation could have identified these issues in advance.

Relatively simple "visual ergonomics" solutions, such as changing the screen resolution, were contraindicated as this degraded the image quality and increased the viewing difficulty of the task. Therefore, the principal recommendations given for solving this visual discomfort included: 
- Engaging a software specialist to investigate the feasibility and cost of modifying the computer interface and

- Establishing a consultative process (e.g. a change management team) to enable worker input and feedback during the redevelopment process.

\subsection{Solving postural discomfort in heavy industry}

A generalist ergonomics consultant was engaged to solve postural issues in the driver's cabin of an overhead travelling crane. The operator sat in a cabin high on a gantry above a storage area and was required to move loads around the yard. To do this, the operator manipulated four controllers which allowed the movement of the gantry, the hoist and attaching and releasing the load. These controllers were slightly forward on either side of the operator's seat. While doing this, the operator viewed:

- The yard and hoist onto which the load was attached. These were below eye level and were visible through a full length glass window.

- A computer monitor which provided information about the job and a CCTV to ensure the load was attached correctly. These were recent additions to the cabin and were located above eye level.

Postural issues identified during the assessment included:

- A mismatch between the height of the controllers and the seat height causing operators to raise their arms.

- Large and awkward reach distances to the controllers from the seated position.

- Awkward arm postures when adjusting the controllers due to the visual requirements and need to reach the foot controls.

During the assessment, an operator who wore bifocal spectacles was also observed adopting exaggerated neck postures while working. This was due to a mismatch between the task design and the spectacle lens design. For example:

- The portion of the spectacle lens used for viewing distance tasks was in the upper portion of the lens, but the task (viewing the yard) was below eye level. This resulted in excessive neck flexion (i.e. tip head forward) to view the yard through the upper portion of the lens.
- The portion of the spectacle lens used for viewing near tasks was in the lower portion of the lens, but the task (viewing the computer monitor and CCTV) was above eye level. This resulted in excessive neck extension (i.e. tip head backwards) to view the monitors through the lower portion of the lens.

The average age of the workforce at this plant was 55 years. Therefore the issue of appropriate prescription eyewear was likely to affect most workers.

The generalist was able to make recommendations to address the initial postural issue (e.g. recommend relocating and reducing the size of the controller boxes within the cabin) but redesigning the cabin or the task to rectify the visual issue was not a feasible option. Instead, it was recommended that further advice be sought from a specialist (visual) ergonomist who had knowledge of the availability and suitability of specific eyewear options and who would also have an appreciation of the task and other ergonomics and organizational issues which may coexist with the visual issue.

\section{Discussion}

One of the roles of a consultant ergonomist is to apply research and knowledge to solve a problem within industry. Visual ergonomics has been used in this paper to illustrate three different aspects of ergonomics consultancy. Case example 1 describes how a company sought specialist ergonomics advice to implement a vision screening program. Case example 2 documents how a visual ergonomics specialist was engaged to solve a problem but after the investigation commenced it was discovered that the solution was to seek alternative specialist advice. Case example 3 illustrates how a generalist ergonomist was engaged to solve a postural problem in an overhead travelling crane, but during the course of the assessment identified issues which required input from a visual ergonomics specialist.

Since many ergonomics problems are multifaceted, it is perhaps unsurprising that both generalist and specialist consultancy roles have evolved in industry. This trend raises issues for consultants, for those seeking consultants advice and for the ergonomics profession. 


\subsection{Challenges for consultants and those seeking their advice}

\section{Is one consultant enough?}

The case examples in this paper depict ergonomists acknowledging the limits of their knowledge and recommending additional input into the problem solving process. This is not an outcome taken lightly, particularly since there are issues of professional pride, an expectation by those employing the consultant that they will be able to provide the solution to a problem, not refer it on to another, and the financial constraints of employing yet another consultant. This is compounded by the perception by some within industry that ergonomics is a sub-specialty of occupational health and safety; these individuals may not expect to encounter further sub-specialties within the ergonomics discipline.

Consultants should be reassured that the IEA recognises that more than one person may be required to solve a presenting problem (e.g. IEA Core Competency 9.2 "Recognises personal and professional strengths and limitations and acknowledges the abilities of others") [7] and the IEA Code of Conduct for Ergonomists: "limit their practice to those areas of ergonomics for which they are qualified by virtue of training and/or experience" [2]). There is perhaps a greater need for this issue to be more openly discussed, particularly within industry, so that it is perceived as a strength rather than as a limitation of the problem-solving process.

\section{Accessing information}

There is an expectation within industry that consultants will provide evidence based solutions [12]. Ergonomics practitioners need to be able to access these resources, either by finding and applying the information themselves (e.g. by keeping abreast of the current scientific literature) or by identifying a person who already has and can apply this knowledge (e.g. a specialist ergonomist).

Public access online databases and search engines are making this task easier for consultants, but there may also be a role for academics to publicize their research finding beyond the boundaries of peerreviewed publications and narrow subject-specialty conferences.

\section{Integrating advice from a variety of sources}

Identifying the best type of consultant to solve a problem, interpret several different opinions and consolidate them into a practical solution is not always an easy process. This fragmentation can be frustrating and costly for the end user (i.e. industry) especially if opinions appear to be contradictory.

Consequently, while consultants may strive to recommend practical and cost effective solutions [18] consistent with IEA core competencies ("Understands the principles of cost-benefit analysis for any ergonomics change") [7] sometimes there is pressure from clients for:

- total solutions which avert the added expense of engaging other consultants or

- easy-to-implement solutions which may not address the cause of the problem.

One way to integrate advice from a variety of sources in a way which can be cost and time effective in the long run is to use a project-management approach e.g. a generalist ergonomist or an employee ergonomist collects advice and recommendations from several specialist ergonomists and uses these to enact the problem solving process. Some consultants currently use this method informally (as demonstrated in the case examples in section 3.1 and 3.3) or formally (e.g. a group of ergonomists form a group who make a joint work proposal).

Such an approach raises challenges for those who engage consultants as they need to determine whether the collective expertise of the group is current, relevant and will address the scope or complexity of the problem.

There are also challenges for consultants who are required to share information with other ergonomists who may otherwise be business competitors; this may be a disincentive for collaboration. The risks of information leakage and loss of competitive advantage have been described as a problem for industry engaging consultants as these consultants may inadvertently or purposefully disclose information gained to other clients [13]. These risks are also true for consultants, particularly specialists, who may have developed particular expertise which allows them to provide a unique service.

On the other hand, collaboration can lead to the integration of specialist knowledge into generalist ergonomics practice, leaving the specialist to solve more complex or specific problems within their domain [11]. Greater mainstream awareness of specialist knowledge could provide opportunities for specialty practice as generalists become cognizant of issues beyond their expertise and recognize that others may be able to supplement their knowledge by joint consultancy (as shown in case example 3, section 3.3). 
Although the IEA code of conduct for ergonomists [2] lists personal qualities such as integrity and recommends that ergonomists respect the privacy of individuals and organizations, there are no explicit statements addressing the risks associated with collaboration. These issues require investigation if collaborative approaches are to be a successful long-term strategy.

\subsection{Challenges for the profession}

\section{Should specialists have generalist training?}

It could be argued that subject matter specialists who are not ergonomists (e.g. other professionals and academics working in disciplines such as healthcare, engineering or psychology) can solve ergonomics problems without having attained generalist ergonomics training because ergonomics is "common sense". However, such an approach can lead to inefficiencies in the problem solving process [19] incorrect conclusions [20] or solutions which do not take into account broader issues within the workplace.

Ergonomics is a holistic discipline. Therefore, it is logical that its practitioners should have an appreciation of the various facets within the profession. This is consistent with the IEA Core Competency "Adopts a holistic view of ergonomics" [7] and is exemplified in case examples 2 and 3 (section 3.2 and 3.3) where the presenting problem had underlying issues which only became apparent after the investigation had commenced. Making an appropriate recommendation (even if this is to seek the advice of another ergonomist) can only be achieved if the ergonomist first on the scene has an awareness of possible contributing factors to a problem. This is true, irrespective of whether the 'first-on-the-scene' ergonomist is a specialist or a generalist.

Generalist training for all ergonomists is one way to prevent fragmentation within the profession caused by the development of special interest groups. Such a strategy would provide a common bond for members of the profession and an appreciation of the skills and expertise of members with different educational backgrounds and work-experience. Similar concerns about fragmentation were voiced within medicine in the 1880s [11] but this has been largely mitigated by providing generalist training to all doctors, who then may progress to become specialists, either by formal education or by work-experience in particular environments or industries.

\section{Conclusion}

Specialist and generalist ergonomics consultants exist within industry and may work together to provide holistic solutions to problems. Since ergonomics problems are frequently multifaceted, ergonomics consultants should have training in all domains of ergonomics, even if they ultimately only practice in one specialty or domain. This is distinct from recognizing academic experts with a specialty in a particular area or an interest in ergonomics but who are not ergonomics practitioners.

There are many issues arising from the development of specialist ergonomics consultancy. These issues need to be discussed by the profession to ensure that ergonomics remains a holistic discipline.

\section{References}

[1] Australian Bureau of Statistics 1321.0 - Small Business in Australia, 2001,

http://www.abs.gov.au/AUSSTATS/abs@,nsf/Lookup/ 1321.0Main+Features12001?OpenDocument. Accessed $18^{\text {th }}$ April 2009.

[2] Code of conduct for ergonomists, International Ergonomics Association, 2006. http://www.iea.cc/01 what/Code $\% 20$ of $\% 20$ Conduct $\% 20$ for $\%$ 20Ergonomists.html Accessed $18^{\text {th }}$ August 2011.

[3] CPEs, Human Factors and Ergonomics Society of Australia. http://www.ergonomics.org.au/membership/cpe.aspx. Accessed $24^{\text {th }}$ August 2011.

[4] Health FAQs, Australian Government Office of the Australian Information Commissioner. http://www.privacy.gov.au/faq/health Accessed $17^{\text {th }}$ August 2011

[5] Indoor Air Quality Handbook: A Practical Guide to Indoor Air Quality Investigations, TSI Incorporated, USA, 2007.

[6] International Ergonomics Association, http://www.iea.cc/what is ergonomist.html Accessed 25th July 2010.

[7] International Ergonomics Association Summary of Core Competencies in Ergonomics: Units and Elements of Competency (2001), International Ergonomics Association. http://www.iea.cc/browse.php?contID=edu competencies. Accessed $2^{\text {nd }}$ August 2011.

[8] Austroads, Assessing fitness to drive: commercial and private vehicle drivers, Austroads Incorporated, Sydney, 2006.

[9] I. Borish, Clinical Refraction Professional Press Books Fairchild Publications, New York, 1985.

[10] R. Bucher and A. Strauss, Professions in Process, American Journal of Sociology, 66 (1961), pp. 325-334.

[11]L. Bulkley, Specialties, and their relation to the medical profession, Journal of the American Medical Association (1884), pp. 651-655.

[12]B. Head, Three lenses of evidence-based policy, The Australian Journal of Public Administration, 67 (2008), pp. 111. 
[13] A. Hoecht and P. Trott, Outsourcing, information leakage and the risk of losing technology-based competencies, European Business Review, 18 (2006), pp. 395-412.

[14] J. Long, The effect of character size on working distance at a computer screen, in V. Blewitt, ed., Ergonomics for Life: At work, home and leisure, Ergonomics Society of Australia Inc, Adelaide, 2000, pp. 40-45.

[15] J. Matarazzo, There is only one psychology, no specialties, but many applications, American Psychologist, 42 (1987), pp. 893-903.

[16] M. Monk and M. Terris, Factors in student choice of general or specialty practice, The New England Journal of Medicine (1956), pp. 1135-1140.
[17] R. North, Work and the Eye, Butterworth Heinemann, Oxford, Great Britain, 2001.

[18] M. Oxenburgh, P. Marlow and A. Oxenburgh, Increasing productivity and profit through health and safety, CRC Press, Boca Raton, Florida, 2004.

[19] M. Stevenson, Notes on the Principles of Ergonomics, Mike Stevenson Ergonomics, Balgowlah, Australia, 1999.

[20] D. Watts, Un-common sense, New Scientist $16^{\text {th }}$ July (2011), pp. 24-25.

[21]D. Werner, Teaching clinical thinking, Optometry and Vision Science, 66 (1989), pp. 788-792. 